\title{
Highlights of a Symposium, Malaria: Where are we Today, Where are we Going?
}

\author{
Rosanne W. Wieten ${ }^{*}, 1$, Michèle van Vugt ${ }^{1}$, Frank van Leth ${ }^{2}$ and Martin P. Grobusch ${ }^{1}$ \\ ${ }^{I}$ Department of Infectious Diseases, Tropical Medicine and AIDS, Division of Internal Medicine, Academic Medical \\ Center, University of Amsterdam, Amsterdam, The Netherlands \\ ${ }^{2}$ Department of Global Health, Academic Medical Center, University of Amsterdam, Amsterdam Institute for Global \\ Health and Development, The Netherlands
}

\begin{abstract}
Malaria continues to pose a major public health threat in endemic areas. However, times are changing, and many investments have been made in recent years into funding of malaria research, the development of more and improved control tools, and applying those to the field. Consequently, there is a renewed interest in going as far as considering the prospects of malaria elimination on a global scale. This goal cannot be reached without optimising and combining biotechnical, economical and social anthropological aspects. A symposium held on 25 January 2011 in Amsterdam, the Netherlands, organised by the Amsterdam Institute for Global Health and Development, the Center for Infection and Immunity Amsterdam and the Academic Medical Center of the University of Amsterdam, focused on malaria and the malERA eradication program, summarizing the state of the art in malaria control and beyond, and offering insight into the various possible ways forward. This manuscript summarizes the information presented and the ensuing discussions.
\end{abstract}

Keywords: Antimalarial drugs, eradication, malaria, symposium, vaccines, vector control.

\section{INTRODUCTION}

Malaria is a major global public health problem, with a huge economic and social impact throughout the endemic areas. On the $25^{\text {th }}$ of January 2011 , a symposium, organised by the Amsterdam Institute for Global Health and Development (AIGHD) [1], the Center for Infection and Immunity Amsterdam (CINIMA) [2] and the Academic Medical Center of the University of Amsterdam (AMC), was held at the AMC, the Netherlands, focusing on malaria and the malERA eradication program, as well as on alternative causes of febrile illness in the tropics. This paper summarizes the information presented and the discussions held, and identifies areas for future research.

In 2009, 225 million cases of clinical malaria and 781,000 malaria related deaths were reported worldwide. About 1.2 billion people (one-fifth of the world's population) were living in areas with high risk of transmission [3]. In 1955, the World Health Organization (WHO) embarked on the Global Malaria Eradication Program (GMEP). This program was discontinued after it was recognised that eradication was not achievable (Box 1). Since then, financial support of malaria programs declined in the 1970s and 1980s [4].

The Malaria Eradication Research Agenda (malERA) initiative was convened in 2008 to define the knowledge base, strategies, and tools required to eradicate malaria from the human population [5].

Address correspondence to this author at the Tropencentrum Academisch Medisch Centrum, Amsterdam, PO 226660, 1100DD Amsterdam, The Netherlands; Tel: 020-5669111 Fax: 020-5664440;

Email: r.w.wieten@amc.uva.nl
In order to reach the final goal of eradication, previous efforts of control and elimination should not be discontinued, but rather implemented in a fitting design to ultimately reach the goal of interruption of transmission.

There are, however, many factors complicating control (and eradication) of malaria. Complicating factors concerning eradication of malaria are: 1) the complexity of the life cycle of the parasite; 2) the complexity of the epidemiology of the disease; 3 ) development of resistance to antimalarial drugs; 4) differences in public health systems and 5) the absence of an adequate malaria vaccine. During the symposium, the speakers addressed several of these problems.

Box 1. Definitions of Control, Elimination and Eradication, adapted from Alonso et al., PLoS Med, 2011

CONTROL: Reduction of disease incidence, prevalence, morbidity or
mortality to a locally acceptable level as a result of deliberate efforts.
ELIMINATION: Reduction to zero of the incidence of locally
transmitted malaria infection in a defined geographical area as a result of
deliberate efforts; continued intervention measures are required to
prevent reestablishment of transmission
ERADICATION: The permanent reduction to zero of the worldwide
incidence of infection.

Marcel Tanner (Swiss Tropical Institute, Basle, Switzerland) gave an overview on the malERA strategies and the developments in malaria elimination/eradication, Peter G. Kremsner (Institute of Tropical Medicine, University of Tuebingen, Germany) provided an overview on current antimalarial drugs and their development while the state-of-the-art current development of vaccines was 
addressed by Pedro Alonso (Centre de Recerca en Salut Internacional de Barcelona, Spain). Martin P. Grobusch (Tropencentrum, Academic Medical Center, University of Amsterdam, the Netherlands) discussed vector control and intermittent preventive treatment strategies; and Tom van der Poll (Department of Infectious Diseases, University of Amsterdam, the Netherlands) closed by broadening the view to other causes of febrile illness in the tropics.

\section{THE BROADER PICTURE: WORKING TOWARDS MALARIA ERADICATION}

Marcel Tanner reiterated that from the previous malaria elimination programme of WHO, the lessons were learnt that (i) politically relatively stable conditions, (ii) good management and effective surveillance, (iii) community awareness and engagement as well as (iv) applied research and considering $P$. vivax were key determinants of success.

The Global Malaria Action Plan (GMAP; RBM 2008) and the malERA process emphasize that when moving from control to elimination/eradication, the conceptual focus shifts from reducing morbidity and mortality to blocking transmission. Thus, a major paradigm shift is necessary on research questions and underlying hypotheses. The malERA process, involving more than 300 scientists and programme managers worldwide, was set up to evaluate available tools and strategies to enhance their use, to address knowledge gaps that need research at biomedical, technological as well as at the health systems level, stimulate the development of new tools and approaches and to promote consensus and partnership in research.

The key to elimination is to take into account the heterogeneities of the current malaria situation. When approaches towards elimination are made, the ecosystemic approaches should be more closely and coherently interlinked with public health approaches. Through better-defined roles and responsibilities, partnership and collaboration can be more effective and will assure that the GMAP is successfully pursued.

\section{ANTIMALARIAL DRUGS}

Peter Kremsner presented an exhaustive overview of old and new drug strategies in the treatment of malaria in his talk on antimalarials. Focusing on $P$. falciparum malaria, he elaborated on the wide range of available antimalarials, with artemisinin combination treatments (ACT) as the main pillar anteceded by light microscopical or 'rapid diagnosis' (by antigen detections tests, RDTs) of those therapies. As opposed to 15 years ago, there is now a pipeline from which new drugs are emerging [6].

The first available antimalarial drug was quinine, which has been in use for over 350 years [7]. Quinine has kept much of its efficacy, although it can no longer be used as a mono-therapy in Southeast Asia due to resistance of $P$. falciparum. Other disadvantages of quinine are severe side effects like: cinchonism (dysphoria, tinnitus, nausea), complex pharmacokinetics and the induction of hypoglycaemia. These adverse effects complicate compliance to the necessary 7 days of administration when the drug is administered as a monotherapy.
Against chloroquine as the model 4-aminoquinoline, $P$. falciparum has mounted widespread resistance in all endemic areas except Central America, and resistance of $P$. vivax is increasingly occurring. However, there is now evidence that in the absence of drug pressure, resistance may reverse $[8,9]$.

Folate antagonists (sulfadoxine-pyrimethamine and proguanil) were developed both as prophylactic and therapeutic agents; however, resistance against these drugs emerged soon afterwards, initially in the 1960's in South America and Asia, later, around the 1980's in Africa where it spread from east to west [10].

Sulfadoxine-pyrimethamine is now rarely used because of resistance, leading to this combination being dropped as replacement first-line therapy for chloroquine in favour of ACTs over the past few years throughout Africa. Because of the low price and the single-dose usage however, it is still used in many African countries both as treatment and as intermittent prophylactic treatment (IPT).

Combining antimalarials is useful since it increases efficacy, shortens the duration of treatment, and decreases the risk of selecting for resistance. Additionally, the chance of mutations creating resistance against several regimes is smaller than against one. Various antimalarial drug combinations are in use; currently, artemisinin combinations are being propagated by the WHO as first line combination.

\section{Artemisinin Combination Therapies (ACTs)}

The WHO currently recommends artemisinin combinations. Artemether derivatives are relatively new drugs that are highly effective. They are combined with other long lasting drugs, in order to reduce the risk of further resistance. Most clinically important artemisinins are metabolised to dihydroartemisinin, in which form they all have comparable antimalarial activity. They are well tolerated, but use in monotherapy has been associated with high incidences of recrudescent infection, suggesting that combination with other antimalarials might be necessary for maximum efficacy.

A recent review comparing artesunate plus amodiaquone, artemeter-lumefantrine, artesunate plus sulfadoxinepyrimethamine, amodiaquone plus sulfadoxinepyrimethamine and dihydroartemisinin-piperaquine for treating uncomplicated $P$. falciparum infection has shown that all ACTs achieved the World Health Organization (WHO) defined threshold of efficacy [11]. ACTs were superior to amodiaquine plus sulfadoxine-pyrimethamine in East Africa. At present, WHO recommends artesunateamodiaquine and arthemether-lumefantrine in Africa.

\section{New Drugs: Dihydroartemisinin-Piperaquine and Fosmidomycin}

A novel combination currently introduced into the ACT portfolio is dihydroartemisinin-piperaquine, which performs well compared to the ACTs in current use [12-14].

Fosmidomycin rapidly eliminates parasites, interfering with the parasite's apicoplast, an organelle interestingly shared with Toxoplasma. When combined with clindamycin, which works synergistically, rapid parasite clearance and high cure rates were achieved [15]. In two studies performed 
at the Albert Schweitzer Hospital in Gabon, good cure rates were reached in children treated for 4 days and more with fosmidomycin-clindamycin and for 3 days and more with fosmidomycin-artesunate. The mean parasite clearance time (PCT) for fosmidomycin-clindamycin was 41 hours; PCT for fosmidomycin-artesunate was 25 hours. The safety of fosmidomycin has been demonstrated in these studies [16].

Marcel Tanner reported that the malERA consultative group on drugs came up with one single key recommendation for drugs in elimination/eradication, i.e. the SERCaP concept of Single Encounter Radical Cure and Prevention [17]. The consultative group has searched for new research questions and knowledge gaps in the field of antimalarial drugs and focused on drugs that could be used for eradication and not, as has been the primary goal in the past, control. Importantly, drugs that completely eliminate malaria parasites from each individual and that block malaria transmission have high research priority.

Radical cure is defined as eliminating all parasites in the patient, and prophylaxis is the prevention of reinfection for some defined period. This should typically be at least one month to outlast the development period of Plasmodium parasites in Anopheles mosquitoes. Both characteristics are essential in eliminating the disease in a population.

Past and present antimalarial drug regimens were often complex and involved multiple administrations. In areas with relatively good health systems this can be successful, however to facilitate administration, $\mathrm{SERCaP}$ is necessary.

Mass drug administration (MDA) schemes have been effective in the past if they encompassed a careful preparatory phase, social mobilisation, improvement of the health care infrastructure and the inclusion of malaria control in comprehensive health care, and the concomitant use of anti-vector measures. Safety becomes an important research issue in MDA, as drugs with better safety profiles are required when administered in whole asymptomatic populations

Knowledge gaps identified by the malERA consultative group for optimizing current drugs relate to the use of 8aminoquinolines and ACTs. Eight-aminoquinolines (a group of quinolone derivatives comprising primaquine, tafenoquine and pamaquine [18]) can kill $P$. vivax dormant liver stages and gametocytes, and primaquine is the only currently licenced 8-aminoquinolone. Antihypnozoite drugs (8aminoquinolones) must remain an important focus of research and profiling of these established drugs therefore entails. Optimal dosing regimens for gametocytocidal efficacy and safety should be investigated as drugs with these characteristics block parasite transmission.

Starting artemisinin resistance in Southeast Asia has begun to threaten the use of ACTs [19, 20]. Research in optimising the use of these drugs is likely to yield high gains at relatively low costs over the next 5-10 years. For example, tests that can detect resistance to artemisinins and ACT partner drugs, rapid glucose-6-phosphate dehydrogenase tests to improve safety of aminoquinoline use and pharmacology studies to optimize dosing regimens of 8aminoquinolines are necessary.
New drugs should attempt to build in strategies to prevent resistance as well, as even in areas with low malaria transmission, resistance can spread rapidly and become fixed.

Importantly, as transmission patterns of malaria vary, so do demands for pharmacokinetic/pharmacodynamic characteristics of new drugs. Setting-specific epidemiology and indications should be taken into account when thinking of desired drug characteristics.

Research into robust and sensitive screening methods is needed to guide drug treatment and prophylaxis. The effectiveness of various screening and administration regimes (e.g. mass screening and treatment, focal screening and treatment and treating all individuals regardless of infection) should be evaluated in specific epidemiological settings and populations.

\section{SULFADOXINE-PYRIMETHAMINE AND INTER- MITTENT PREVENTIVE TREATMENT (IPT)}

Martin Grobusch addressed the concept of IPT. Particularly in areas with high malaria endemicity and where sulfadoxine-pyrimethamine resistance rates are still acceptable (SP is amongst currently available drugs favoured for its long half-life but alternative drug combinations are investigated) this additional group of intervention tools is considered useful, particularly as they come at low cost and as they are combinable with established Public Health care measures such as Antenatal Care and the Expanded Programme of Immunization.

Intermittent prophylactic treatment is used in pregnancy (IPTp), infants (IPTi) and in older children (IPTc), particularly in areas with seasonal transmission (sIPT). By administration of chemoprophylaxis at regularly scheduled intervals, regardless of the plasmodial infection state, IPT programs aim at reducing malaria morbidity and mortality. The exact mechanism of IPT is not clear, however it is assumed that it works primarily by prevention and additionally provides a development of protective antibodies due to subclinical infection, as IPT does not entirely prevent malaria episodes [21].

In pregnancy, IPT diminishes the effects of malaria on both foetus and mother. In a Cochrane review from 2006 it is concluded that IPT reduces antenatal parasite prevalence and placental malaria. Also, IPT has positive effects on birth weight (up to $35 \%$ of preventable low birth weight is caused by malaria in endemic areas) and on perinatal death in lowparity women [22]. The WHO currently recommends the use of IPT in pregnant women in highly malarious areas, with the exception of subjects with established HIV seropositivity. Sulfadoxine-pyrimethamine has a low cost, high availability, easy delivery and high acceptability profile and is therefore the drug of choice in countries where efficacy remains good. In countries where resistance rises, other regimens require evaluation (such as amodiaquone with or without SP, artesunate and SP, chlorproguanildapsone with and without artesunate and artemetherlumefantrine) [23].

Trials investigating IPTi in Tanzania with SP and amodiaquine in infants revealed greater than 50 and $60 \%$ reductions in clinical malaria and anemia, respectively. 
In 2009, a review by the IPT in infants (IPTi) consortium analyzed six trials conducted in Africa evaluating the efficacy and safety of IPTi with sulfadoxine-pyrimethamine, including a total of 7930 infants. All trials were randomized, double blind, placebo controlled. It was concluded that IPTi is safe and does not interact with EPI vaccines. Importantly, IPTi had a pooled protective efficacy of $30.3 \%$ (95\% CI 19.8-39.4, $\mathrm{p}<0.0001)$ [24].

Further analysis concluded that anemia was reduced by $15 \%$, malaria admissions were reduced by $38 \%$ and all-cause admissions were reduced by $23 \%$. IPTi was found to be deliverable by the existing health system and is affordable and acceptable [25]. It was pointed out that protective efficacy is not correlated to failure rates of SP; therefore SP probably has a different route of action in prevention and treatment.

The WHO now recommends implementation of IPTi alongside EPI programmes in countries in sub-Saharan Africa with moderate-to-high malaria transmission (where annual entomological inoculation rates are $\geq 10$ ) and where parasite resistance is not high (defined as a prevalence of the pf dhps 540 mutation of $\leq 50 \%$ ).

IPT in children up to the age of 5 and in areas of high seasonal transmission is currently under scrutiny. The delivery of IPT via EPI programs is effective for infants; however, for children, the most effective way of delivery is uncertain. Therefore, various ways of administration are being investigated. For example, administration via village health workers showed to be effective [22]. Long acting ACTs may also make important reductions in malaria transmission [21]. Delivery systems that reach individuals most exposed to malaria should be developed for these areas of high seasonal transmission.

\section{VACCINES}

Pedro Alonso discussed current issues in the field of vaccine development. Vaccines in general are, on a global scale, undisputedly the most successful infectious diseases control tools and can be used even in areas with weak health systems. However, malaria is an immunologically challenging infection. First, there are many uncertainties concerning the human immune response against the malaria parasites. Previous funding of research in this field has been limited. Second, malaria parasites exhibit immune evasion strategies. There is marked antigenic polymorphism and variation between parasites, as well as stage specific antigen expression within parasites.

After a deterioration of the malaria situation in the 1980 's, particularly after the development of chloroquine resistance, the SPf66-vaccine was received enthusiastically. This vaccine was extensively studied. It was safe and immunogenic, however results of the efficacy trials varied [27]. Studies from that time gave insight in challenges; for example that there is a lack of surrogate markers for protection: IgG antibodies do not correlate with clinical protection or inhibition of parasite growth in vitro [28]. Also, there is a lack of animal models.

Since the introduction of the SPf66-vaccine, attitudes towards malaria control have changed. Funding has become available increasingly and reinvigorated vaccine research.

The malERA consultative group of vaccines introduced the concept of "vaccines that interrupt malaria transmission" (VIMT) [29]. This concept includes not only classical transmission blocking vaccines which target the sexual and mosquito stages, but also pre-erythrocytic and asexual stage vaccines. VIMTs should be effective against $P$. vivax and $P$. falciparum, suitable for all age groups, pregnant women and immunecompromised persons and they should impact transmission.

Current Status in Antimalarial Drug Development:

ACTs are currently recommended as first line treatment; however, resistance is emerging, threatening effective use of ACTs

Eight-aminoquinolines are the only drugs in use with hypnozoitocidal properties

Intermittent Prophylactic Treatment with SP is currently in use in areas with high malaria endemicity and acceptable levels of SP resistance

Current Challenges in Antimalarial Drug Development:

To develop tests that can detect resistance to artemisinins and ACT partner drugs

To maintain the development pipeline of new drugs

- For first-line treatment of P. falciparum

- For malaria eradication:

- Killing or preventing development of gametocytes

- Blocking sporozoites in the mosquito

- Curing liver stages of $P$. vivax and $P$. ovale

- Single encounter radical cure dosing, sustained or pulsed formulations

To optimize the use of currently available drugs:

- Dose regimens for gametocytocidal and anti-relapse efficacy and safety

- Glucose-6-phosphate dehydrogenase (G6PD) test to improve safety of 8-aminoquinolones

\section{Research Topics on Antimalarial Drugs:}

Mechanisms of resistance and pharmacological strategies to deter resistance

Fundamental research of liver and sexual stage biology (both in host and in mosquito), for developing drugs for eradication

Strengthened focus on P.vivax: in vitro culture and study of hypnozoites

The feasibility of other drugs potentially used in IPT 
Pedro Alonso explained that the pre-erythrocytic vaccine decreases probability of a person getting infected following an infectious mosquito bite. Blood stage vaccines decrease symptoms and decrease the length of time or level of infectiousness. Mosquito stage vaccines decrease the number of infectious mosquitoes. Importantly, all three are potentially useful if they have a direct impact on the survival of parasites.

The RTS,S vaccine is the leading vaccine candidate. It is a pre-erythrocyte candidate vaccine that consists of hepatitis $\mathrm{B}$ surface antigen and the surface antigen from the circumsporozoite, combined with an adjuvant (AS02/AS01). In phase II field trials in the Gambia [30] and Kenya [31], RTS,S vaccination conferred short-lived protection against malaria infection in approximately $35 \%$ of adults. It has recently been shown in phase II field studies in Kenyan, Mozambiquan and Tanzanian children, that $30-50 \%$ were protected from malaria after immunization with RTS,S [3235].

The overall conclusions that can be drawn from the phase II trials with the RTS,S vaccine are that there is a trend towards higher efficacy against severe forms of disease as well as on mortality. Protection in a cohort with a higher attack rate by mosquitoes appeared considerably shorter and there was evidence of waning immunity in young infants. The RTS,S/AS vaccine might act as a partially protective vaccine, lowering, but not blocking, multiplicity and new infections. Due to this mechanism, called a 'leaky vaccine', natural immunity may be acquired [36]. Currently, it is undergoing phase III clinical trials in African children. The MAL050 study investigating AS01 regimens with a follow up of 19 months show vaccine efficacy of up to $60 \%$ (P. Alonso, unpublished results).

The WHO provides a summary of global malaria vaccine projects at advanced pre-clinical and clinical stages; there are many combinations with other vaccines in the pipeline, against antigens of all stages of the malaria parasite [6].

Mathematical models visualizing parameters influencing malaria transmission show that in order to reduce the case reproduction rate, which is, in short, the number of secondary cases arising from a single case in a fully susceptible human population, not only vaccines, but also vector control/bed nets and antimalarial drugs are necessary. Especially since none of the vaccines currently in development will be close to $100 \%$ efficacy. This is shown in a review of the global malaria vaccine pipeline, in which all the current available vaccines aim at partial protection [6].
By 2015, a first generation malaria vaccine that has protective efficacy of more than $50 \%$ against severe disease and death and that induces protective efficacy for a period longer than 1 year will most likely be commercialized. The mid-term strategic goal of the malERA group is to have a licensed malaria vaccine that has a protective efficacy of more than $80 \%$ against clinical disease and lasts longer than four years by 2025 . The challenges are the long period for a first generation vaccine to get licenced (approximately $1 / 4^{\text {th }}$ of a century) and the high costs for vaccine development (more that 500 million USD).

\section{VECTOR CONTROL}

Martin Grobusch further discussed the need for increased vector control efforts. Vector-based control strategies target an essential player in the transmission process. They address a step that precedes infection and that is not dependant on human compliance.

Since the discovery of Robert Ross in 1897 that malaria is transmitted by mosquitoes, vector control strategies have been an important part of malaria control programmes. Among these strategies are environmental management, insecticide treatment, and molecular entomological approaches [37]. Environmental management (e.g. draining wetlands and using larvivorous fish) varies with the variation of the ecological habitat of the vector.

Pesticides, previously abandoned because of resistance of vectors and public rejection due to ecological impact, are currently still used for insecticide treated bed nets (ITN) and indoor spraying. Both impregnated bed nets and indoor spraying need to be available frequently and at low costs in order to be effective [38-40].

A Cochrane Review concluded that insecticide treated bed nets are highly effective in reducing childhood morbidity and mortality from malaria; however financial, technical and operational inputs are required for effective universal use [41].

Indoor residual spraying (IRS) is highly efficacious; however, there are insufficient trials to quantify the effects of IRS in areas with different malaria transmission, and few trials comparing ITNs and IRS [42].

Mathematical models on effectiveness of ITNs and IRS suggest that high coverage is necessary to achieve interruption of transmission, and that the combination of the two methods with DDT used simultaneously can interrupt transmission. However, these models contain many assumptions and need adaptations based on more field data [43].

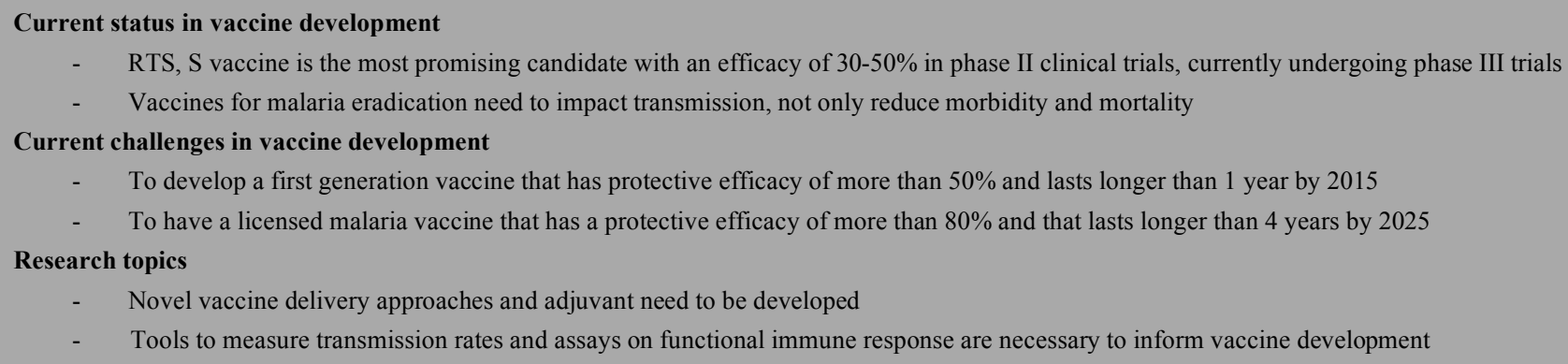


Sequencing of the $A$. gambiae genome has provided insight into the vector's biological processes. Identification of new targets that can be used to disrupt or exploit the vector's biological process is followed by the development of new approaches for controlling malaria. The predominant focus of research is on inhibition of development of the parasite during its sexual stage transition (gametocytogenesis) and the midgut penetration of the host (by ookinetes) [37].

Also, vaccine development (in terms of including an 'altruistic' transmission-blocking component in addition to vaccinee-protecting antigens), as discussed above, and various routes of behavioural modification are currently of increased interest. For example, many molecular components that interact with odorants have been identified recently. However, numerous questions of for example the way odorant receptors, odorant receiving proteins and olfactory receptor neurons in the mosquito respond positively or negatively to certain odors remain unanswered [37]. Insight in the function of these receptors might offer effective tools in controlling mosquito behaviours via chemosensory repellents and attractants. The same accounts for trapping mosquitoes through light attraction, as mosquitoes rely on photo-sensory mechanisms and their circadian rhythms for proper biological functioning.

Exploiting mosquito immune response is a potential mechanism in limiting Plasmodium infection and transmission. Several proteins (Toll, IMD, Jak/Stat) that play a role in the mosquito's innate immune system preventing infection by Plasmodium spp. have been identified. Genetic modification boosting the immune response of mosquitoes and the introduction and stable transmission of inhibitory bacteria in mosquitos might both be ways of controlling malaria transmission [44].

Sterile insect technique (SIT) sterilizes male mosquitos by ionizing radiation before mass release [45]. Irradiation has proved to be a successful, safe, and accepted way to sterilize large numbers of insects. Striking the optimal balance between $100 \%$ sterility but decreased competitiveness and partial sterility but higher competitiveness is necessary for determining the optimal radiation dose. Studies analyzing these aspects in a (semi-) field setting are needed.

Recently, Wen Kilama et al. voiced the malaria community's need to consider ethical aspects with regard to field trials of vector interventions [46]. As there is lack of experience with many of the promising candidate tools, such as the genetically modified mosquitos and biocontrol agents, caution must be exercised before they are introduced in the field. Phase 3 trials, which are undertaken on vulnerable civilian populations, pose dilemmas, especially on individual and community safety. Wen Kilama concludes that new tools assessed in these trials should be safe, beneficial, efficacious, effective and acceptable to large populations in the long and short term. Also, the burdens and benefits of research should be equally distributed.

Martin Grobusch concluded by stressing that integrating vector control efforts will have to feature prominently in the quest for intensified, successful malaria control in the years to come.

\section{Sepsis}

Malaria and its role in morbidity and mortality in Africa were put into perspective by Tom van der Poll in this presentation entitled 'Thinking beyond malaria: bacterial disease as an important cause of febrile illness in the tropics'. WHO figures show that of 11.2 million deaths in Africa, $7.2 \%$ are caused by malaria; an increasing wealth of research highlights the importance of preceding malaria treatment by an adequate diagnostic tool in order to avoid over-treatment, and not to miss opportunities to timely establish a correct alternative diagnosis, as HIV/AIDS and its opportunistic infections $(14.7 \%)$, respiratory tract infections (14.7\%), diarrhoea (8.9\%) and TB (3.6\%) are also important contributors to mortality (Fig. 1).

Of a worldwide number 10.4 million deaths of children under 5, 4.7 million (45\%) occur in Africa, and an additional 3.1 million deaths $(30 \%)$ occur in the Southeast Asian region. Malaria as a cause of death occurs almost exclusively in the African region, where it causes $16 \%$ of morbidity in children aged $0-4$. The predominant causes of morbidity are perinatal conditions, diarrhoeal diseases and respiratory diseases [47].

Pneumonia kills an estimated 1,6 million children per year, accounting for $18 \%$ of all deaths among children $<5$ years of age. Pneumonia causes more deaths than HIV/AIDS, tuberculosis and malaria combined [47].

In 2010, Reddy and colleagues conducted a literature review including 22 studies performed in Africa from 1984 to 2006 with a total of 50,296 patients. Of all adults $(15,166)$ and children $(43,130)$ included, respectively $2051(13.5 \%)$ and $3527(8.3 \%)$ had positive blood cultures. 1643 (29.1\%) non-malaria bloodstream infections were due to Salmonella enterica, the most common isolate in adults, and 1031 $(18.3 \%)$ were due to Streptococcus pneumoniae, the most 


\section{Causes of death in Africa, 2004}

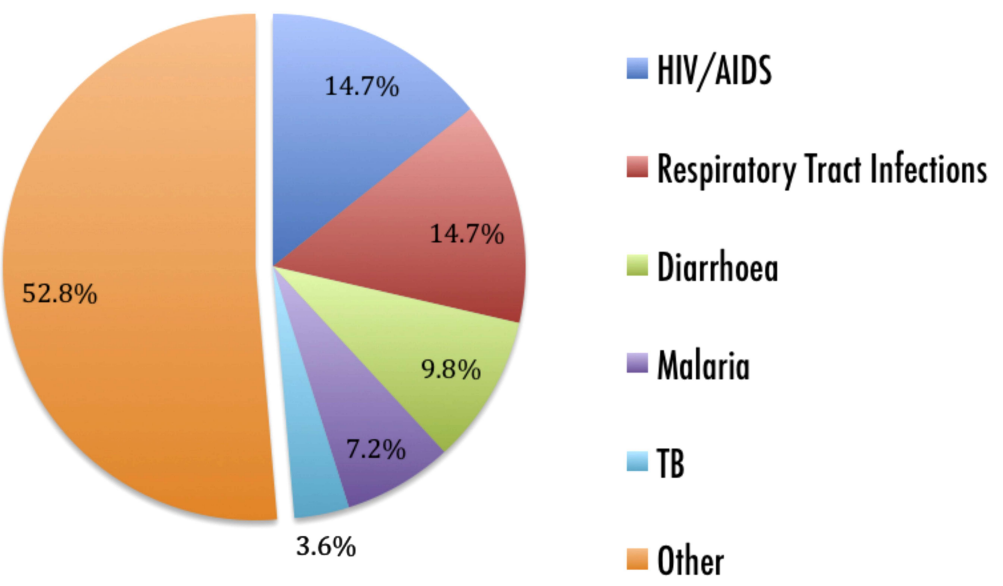

Fig. (1). Causes of death in Africa, after WHO Global burden of disease report, 2004. Total deaths: 11.2 million.

common isolate in children. Other common causes included Staphylococcus aureus (531 infections; 9.5\%) and Escherichia coli $(412 ; 7.3 \%)$. HIV infection was associated with any bloodstream infection, particularly with Salmonella enterica and Mycobacterium tuberculosis bacteria complex bacteraemia. It was shown that bloodstream infections are common in Africa and associated with high mortality. In nine studies involving 11,814 patients where both bacteremia and parasitemia were taken into account, $6.5 \%$ of patients who had parasitemia also had bacteremia.

In order to improve sepsis survival rates, the Surviving Sepsis Campaign, administered by the Society of Critical Care Medicine (SCCM), the European Society of Intensive Care Medicine (ESICM), and the International Sepsis Forum (ISF), was set up in 2002. By systematic literature review and conferences to reach international consensus, protocols for sepsis interventions in low-income countries have been set up. Bundles for low-income countries include laboratory testing (malaria blood smears, bacteria cultures and rapid HIV testing) and therapy (early administration of antibiotics, intravenous fluids and surgery). Rapid administration of antibiotics is of crucial importance in preventing death caused by sepsis [48].

As the prevalence of bacteremia exceeds the prevalence of malaria among patients admitted in some regions, it is important for clinicians to be aware of non-malarial bloodstream infections in febrile patients.

\section{CONCLUSIONS}

This symposium provided an overview of the areas on which the program of elimination/eradication of malaria is going to focus. In order to reach the goal of eliminating/eradicating malaria, an integrated approach using combinations of vaccines, drugs, insecticide treated nets, targeted vector control and health systems interventions that are tailored to a given endemic setting is necessary. Existing tools are not sufficient, hence the focus on research gaps as undertaken by the MalERA-process should accompany the GMAP. Practical insight was provided in the new and necessary approaches for surveillance-response-systems as well as for case management.
Besides effectively using rapid diagnostic tests and clinical exams, clinicians, need to remain aware of the broad and other bacterial causes of febrile illness in the tropics and to have protocols on effective treatment strategies for either infection.

Thinking about control, elimination, or eradication of malaria must be within a cross-cutting context, including a proper approach to health system strengthening. The newly founded Amsterdam Institute for Global Health and Development is committed to contributing to the global goals and the required approaches since its mission is to provide sustainable solutions to major health problems across our planet, by forging synergies between disciplines, health care delivery, research and education.

\section{ACKNOWLEDGEMENTS}

We thank Professors Marcel Tanner (Swiss Tropical Institute, Basle, Switzerland), Pedro Alonso (Centre de Recerca en Salut Internacional de Barcelona, Spain) and Tom van der Poll as well as Dr. Peter de Vries (both Department of Infectious Diseases, Tropical Medicine and AIDS, Academic Medical Center, Amsterdam, the Netherlands), for thoughtful comments on the manuscript.

\section{REFERENCES}

[1] AIGHD. Amsterdam Institute for Global Health and Development. 2010-2011 Available from: http://aighd.org/; [Date of access 21st May 2011].

[2] CINIMA. Center for Infection and Immunity Amsterdam. Available from: http://www.amc.uva.nl/index.cfm?sid=665. [Date of access: 31st May 2011].

[3] Alonso PL, Brown G, Arevalo-Herrera M, Binka F, Chitnis C, Collins F. A research agenda to underpin malaria eradication. PLoS Med 2011; 8(1): e1000406.

[4] Najera JA, Gonzalez-Silva M, Alonso PL. Some lessons for the future from the Global Malaria Eradication Programme (19551969). PLoS Med 2011; 8(1): e1000412.

[5] The Global Malaria Action Plan - for a malaria free world. 2008 Available from: http://www.rollbackmalaria.org/gmap/gmap.pdf [Date of access: 31st May 2011].

[6] WHO. Malaria Vaccine Rainbow Tables. 201121 May 2011]; Available from: http://www.who.int/vaccine research/links/Rain bow/en/ [Date of access: 21st May 2011].

[7] Honigsbaum M. The Fever Trail. $1^{\text {st }}$ ed. London: MacMillan 2001. 
[8] Laufer MK, Thesing PC, Eddington ND, et al. Return of chloroquine antimalarial efficacy in Malawi. N Engl J Med 2006; 355: 1959-66.

[9] Ursing J, Kofoed PE, Rodrigues A, et al. Similar Efficacy and tolerability of double-dose chloroquine and artemetherlumefantrine for treatment of Plasmodium falciparum infection in Guinea-Bissau: A randomized trial. J Infect Dis 2011; 203: 109-16.

[10] Wongsrichanalai C, Miller RS. Malaria rapid tests: a public health perspective. Lancet 2002; 359(9319): 1781.

[11] Sinclair D, Zani B, Donegan S, Olliaro P, Garner P. Artemisininbased combination therapy for treating uncomplicated malaria. Cochrane Database Syst Rev 2009(3): CD007483.

[12] Nambozi M, Van Geertruyden JP, Hachizovu S, et al. Safety and efficacy of dihydroartemisinin-piperaquine versus artemetherlumefantrine in the treatment of uncomplicated Plasmodium falciparum malaria in Zambian children. Malar J 2011; 10: 50.

[13] Mayxay M, Keomay S, Khanthavong M, Souvannasing P, Stepnewska K, Khomthilath T. A phase III, randomized, noninferiority trial to assess the efficacy and safety of dihydroartemisinin-piperaquine in comparison with artesunatemefloquine in patients with uncomplicated Plasmodium falciparum malaria in southern Laos. Am J Trop Med Hyg 2010; 83(6): 12219.

[14] Valecha N, Phyo AP, Mayxay M, et al. An open-label, randomised study of dihydroartemisinin-piperaquine versus artesunatemefloquine for falciparum malaria in Asia. PLoS One 2010; 5(7): e11880.

[15] Borrmann S, Issifou S, Esser G, et al. Fosmidomycin-clindamycin for the treatment of Plasmodium falciparum malaria. J Infect Dis 2004; 190(9): 1534-40.

[16] Borrmann S, Adegnika AA, Matsiegui PB, et al. Fosmidomycinclindamycin for Plasmodium falciparum Infections in African children. J Infect Dis 2004; 189(5): 901-8.

[17] Alonso PL, Djimde A, Kremsner P, et al. A research agenda for malaria eradication: drugs. PLoS Med 2011; 8(1): e1000402.

[18] Kaur K, Jain M, Reddy RP, Jain R. Quinolines and structurally related heterocytes as antimalarials. Eur J Med Chem 2010; 45(8): 3245-64.

[19] Noedl H, Socheat D, Satimai W. Artemisinin-resistant malaria in Asia. N Engl J Med 2009; 361(5): 540-1.

[20] Dondorp AM, Nosten F, Yi P, et al. Artemisinin resistance in Plasmodium falciparum malaria. N Engl J Med 2009; 361(5): 45567.

[21] Grobusch MP, Egan A, Gosling RD, et al. Intermittent preventive therapy for malaria: progress and future directions. Curr Opin Infect Dis 2007; 20(6): 613-20.

[22] Garner P, Gulmezoglu AM. Drugs for preventing malaria in pregnant women. Cochrane Database Syst Rev 2006; 4: CD000169.

[23] Newman RD, Hailemariam A, Jimma D, et al. Burden of malaria during pregnancy in areas of stable and unstable transmission in Ethiopia during a nonepidemic year. J Infect Dis 2003; 187(11): 1765-72.

[24] Aponte JJ, Schellenberg D, Egan A, et al. Efficacy and safety of intermittent preventive treatment with sulfadoxine-pyrimethamine for malaria in African infants: a pooled analysis of six randomised, placebo-controlled trials. Lancet 2009; 374(9700): 1533-42.

[25] Cairns M. Modelling the protective efficacy of alternative delivery schedules for intermittent preventive treatment of malaria in infants and children. PLoS One 2011; 6(4): e18947.

[26] Bojang KA, Akor F, Conteh L, et al. Two strategies for the delivery of IPTc in an area of seasonal malaria transmission in the Gambia: a randomised controlled trial. PLoS Med 2011; 8(2): e1000409.
[27] Graves P, Gelband H. Vaccines for preventing malaria (SPf66). Cochrane Database Syst Rev 2006; 2: CD005966.

[28] Ferreira M. Malaria vaccine trials: the missing qualitative data. Immunol Cell Biol 1996; 74(4): 301-5.

[29] Alonso PL, Ballou R, Brown G, et al. A research agenda for malaria eradication: vaccines. PLoS Med 2011; 8(1): e1000398.

[30] Bojang KA, Milligan PJ, Pinder M, et al. Efficacy of RTS,S/AS02 malaria vaccine against Plasmodium falciparum infection in semiimmune adult men in The Gambia: a randomised trial. Lancet 2001; 358(9297): 1927-34.

[31] Polhemus ME, Remich SA, Ogutu BR, et al. Evaluation of RTS,S/AS02A and RTS,S/AS01B in adults in a high malaria transmission area. PLoS One 2009; 4(7): e6465.

[32] Abdulla S, Oberholzer R, Juma O, et al. Safety and immunogenicity of RTS,S/AS02D malaria vaccine in infants. N Engl J Med 2008; 359(24): 2533-44.

[33] Bejon P, Lusingu J, Olotu A, et al. Efficacy of RTS,S/AS01E vaccine against malaria in children 5 to 17 months of age. $\mathrm{N}$ Engl $\mathrm{J}$ Med 2008; 359(24): 2521-32.

[34] Olotu A, Lusingu J, Leach A, et al. Efficacy of RTS,S/AS01E malaria vaccine and exploratory analysis on anti-circumsporozoite antibody titres and protection in children aged 5-17 months in Kenya and Tanzania: a randomised controlled trial. Lancet Infect Dis 2011; 11(2): 102-9.

[35] Sacarlal J, Aide P, Aponte JJ, et al. Long-term safety and efficacy of the RTS,S/AS02A malaria vaccine in Mozambican children. J Infect Dis 2009; 200(3): 329-36.

[36] Moorthy VS, Ballou WR. Immunological mechanisms underlying protection mediated by RTS,S: a review of the available data. Malar J 2009; 8: 312.

[37] Ramirez JL, Garver LS, Dimopoulos G. Challenges and approaches for mosquito targeted malaria control. Curr Mol Med 2009; 9(2): 116-30.

[38] Shiff C. Integrated approach to malaria control. Clin Microbiol Rev 2002; 15(2): 278-93.

[39] Curtis CF, Mnzava AE. Comparison of house spraying and insecticide-treated nets for malaria control. Bull World Health Organ 2000; 78(12): 1389-400.

[40] WHO. Insecticide-treated mosquito nets: a WHO position statement 2007. Available from: http://www.who.int/malaria/public ations/atoz/itnspospaperfinal.pdf [Date of access: 31st May 2011].

[41] Lengeler C. Insecticide-treated bed nets and curtains for preventing malaria. Cochrane Database Syst Rev 2004; 2: CD000363.

[42] Pluess B, Tanser FC, Lengeler C, Sharp BL. Indoor residual spraying for preventing malaria. Cochrane Database Syst Rev 2010; (4): CD006657.

[43] Chitnis N, Schapira A, Smith T, Steketee R. Comparing the effectiveness of malaria vector-control interventions through a mathematical model. Am J Trop Med Hyg 2010; 83(2): 230-40.

[44] Cirimotich CM, Dong Y, Garver LS, Sim S, Dimopoulos G. Mosquito immune defenses against Plasmodium infection. Dev Comp Immunol 2010; 34(4): 387-95.

[45] Helinski ME, Parker AG, Knols BG. Radiation biology of mosquitoes. Malar J 2009; 8(Suppl 2): S6.

[46] Kilama WL, Ethical perspective on malaria research for Africa. Acta Trop 2005; 95(3): 276-84.

[47] WHO. The Global Burden of Disease - 2004 Update. Available from:

http://www.who.int/healthinfo/global_burden_disease/GBD_report _2004update_full.pdf [Date of access: 21st May 2011].

[48] Kumar A, Roberts D, Wood KE, et al. Duration of hypotension before initiation of effective antimicrobial therapy is the critical determinant of survival in human septic shock. Crit Care Med 2006; 34(6): 1589-96. 